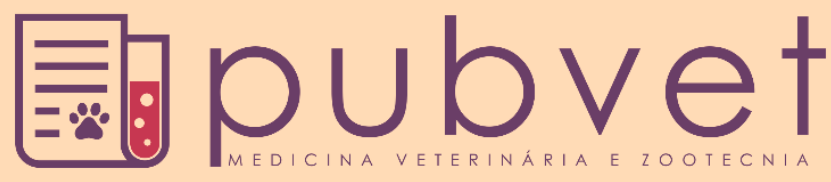

https://doi.org/10.31533/pubvet.v14n12a704.1-8

\title{
Esplenectomia total em cão apresentando dois baços: Relato de caso
}

\author{
Karolliny Thais Rodrigues de Souza ${ }^{* \bullet}$, Marcelo Alves Herdy ${ }^{2} \bullet$, Liliane Maria Valentim Willi \\ Monteiro $^{20}$, Fabiana Batalha Knackfuss ${ }^{2} \bullet$
}

${ }^{1}$ Graduando(a) na Universidade do Grande Rio, Medicina Veterinária. Duque de Caxias -RJ Brasil

${ }^{2}$ Professor(a) na Universidade do Grande Rio, Medicina Veterinária. Duque de Caxias -RJ Brasil.

*Autor para correspondência,E-mail: karollinysouza@unigranrio.br

Resumo. A esplenectomia total tem sua constância na clínica cirúrgica em alterações como neoplasias esplênica, torções (estômago ou baço) ou trauma grave que acarrete uma hemorragia que não possa ser cessada, assim levando ao paciente risco de morte. $\mathrm{O}$ uso dessa técnica traz resultados eficazes em cães, quando associada a distúrbios de caráter hemolítico onde o uso da terapia não se mostra eficaz. Possui curso clínico relacionado a distensão, podendo ser ocasionada por massas, esplenomegalia generalizada ou até mesmo por hemorragia intra-abdominal gerado por trauma. O objetivo deste trabalho foi relatar um caso de esplenectomia total em um cão apresentando dois baços. Um canino macho, não castrado, sem raça definida (SRD), três anos e oito meses de idade, com queixa de atropelamento apresentando sangramento mandibular. Na ultrassonografia foi visto duas estruturas de parênquima e forma igual com descontinuidade de bordo em lobo cranial e heterogeneidade sugestivo de ruptura, além disso, contatou-se presença de discreta quantidade de líquido livre abdominal assemelhando-se a hemorragia. Foi instituído procedimento cirúrgico sendo realizada abertura de cavidade abdominal onde se constatou a presença de dois baços e regeneração de tecido esplênico em região de mesentério. Sendo estabelecida terapia cirúrgica utilizando-se o método de esplenectomia total, onde se obteve sucesso apesar das alterações vistas no pós-operatório, tornando-se possível acompanhar a recuperação do paciente junto à terapêutica instituída.

Palavras chave: canino, dois baços, esplenectomia total, ruptura

\section{Total splenectomy in a dog with two spleens: Case report}

Abstract. Total splenectomy has its constancy in the surgical clinic in alterations such as splenic neoplasms, sprains (stomach or spleen), or severe trauma that causes unstoppable hemorrhaging, thus leaving the patient at risk of death. The use of this technique brings effective results in dogs when associated with disorders of hemolytic character where the use of therapy is not effective. There is clinical development related to distension, which can be caused by masses, generalized splenomegaly, or even by intra-abdominal hemorrhaging caused by a lesion. The aim of this study was to report a case of total splenectomy in a dog with two spleens. A male, non-neutered, mixed breed (SRD) dog, three years and eight months old, with a hit-and-run complaint and mandibular bleeding. On ultrasonography, two structures of parenchyma, equal in shape, were seen with the discontinuity of the edge in the cranial lobe and heterogeneity suggestive of rupture, in addition to the presence of a discrete amount of free abdominal fluid resembling hemorrhaging. A surgical procedure was instituted and the abdominal cavity was opened, where the presence of two spleens and splenic tissue regeneration was found in the mesentery region. Surgical therapy was established using the total splenectomy method, being successful despite the changes seen in the postoperative period, making it possible to monitor the patient's recovery with the instituted therapy.

Keywords: canine, two spleens, total splenectomy, rupture 


\title{
Esplenectomía total en un perro con dos bazos: reporte de un caso
}

\begin{abstract}
Resumen. La esplenectomía total tiene su constancia en la clínica quirúrgica en alteraciones como neoplasias esplénicas, esguinces (estómago o bazo) o traumatismos graves que causan una hemorragia que no se puede detener, lo que lleva al paciente al riesgo de muerte. El uso de esta técnica trae resultados efectivos en perros, cuando se asocia con trastornos de carácter hemolítico donde el uso de la terapia no es efectivo. Tiene curso clínico relacionado con la distensión, que puede ser causada por masas, esplenomegalia generalizada o incluso por hemorragia intraabdominal generada por un trauma. El objetivo de este estudio fue informar un caso de esplenectomía total en un perro con dos bazos. Un perro macho, no castrado, de raza mixta (SRD), de tres años y ocho meses de edad, con una queja de golpe y fuga con sangrado mandibular. En la ecografía, se observaron dos estructuras de parénquima e igual forma con discontinuidad del borde en el lóbulo craneal y heterogeneidad sugestiva de ruptura, además, se encontró la presencia de una cantidad discreta de líquido abdominal libre parecido a hemorragia. Se instituyó un procedimiento quirúrgico y se abrió una cavidad abdominal, donde se encontró la presencia de dos bazos y la regeneración del tejido esplénico en la región del mesenterio. La terapia quirúrgica se estableció utilizando el método de esplenectomía total, donde tuvo éxito a pesar de los cambios observados en el período postoperatorio, lo que permitió controlar la recuperación del paciente con la terapia instituida.
\end{abstract}

Palabras clave: canino, dos bazos, esplenectomía total, ruptura

\section{Introdução}

O baço é o maior órgão linfoide secundário do organismo, no cão é o único órgão intermédio na circulação sanguínea, rico em células fagocitárias, com importante função de proteger o organismo contra microrganismos que possam estar na corrente sanguínea, estabelecendo seu papel na resposta imunitária (Junqueira \& Carneiro, 2013).

Anatomicamente, o baço se encontra na parte cranial esquerda abdominal, englobado ao omento, unido a curvatura maior do estomago pelo ligamento gastro-esplênico. Possui forma variável entre espécies, sendo um órgão falciforme, delgado e longo, e nos cães a porção ventral é mais desenvolvida (Junqueira \& Carneiro, 2013; Kisseberth \& McEntee, 2008). Devido a posição anatômica e funcional, este torna-se alvo de múltiplas desordens, como neoplásicas e não-neoplásicas (Stedile, 2007).

A esplenomegalia é o sinal clínico mais considerável para suspeita de doença esplénica (Aster, 2005). Traumas abdominais têm uma incidência grande, causando lesões que na maioria das vezes são de caráter emergencial devido a causas como atropelamento ou outro acidente (Petroianu et al., 2004), gerando assim o surgimento de ruptura esplênica. Frequentemente a esplenectomia total tem sua constância na clínica cirúrgica em alterações como neoplasias esplênica, torções (estômago ou baço) ou trauma grave que acarrete uma hemorragia que não possa ser cessada, assim levando ao paciente risco de morte (Fossum, 2014).

Uma desordem incomum é a presença de dois baços. Em humanos, é descrito que de 6 a 10\% da população possui dois baços e que essa condição não traz problemas ao indivíduo (Kaulitzki, 2018), sendo este dado ainda não verificado em animais.

Desde forma, o objetivo deste trabalho foi evidenciar essa desordem incomum e o sucesso obtido na técnica cirúrgica, visto que na rotina clínica são raros os casos de pacientes com dois órgãos esplênicos, ainda que a esplenectomia total por ruptura de baço seja bem descrita em literatura, a descrição do mesmo pode contribuir para a orientação de futuros casos.

\section{Relato de caso}

Foi atendido no Hospital Escola de Medicina Veterinária Unigranrio em Duque de Caxias (RJ), um canino macho, não castrado, SRD, de três anos e oito meses de idade, com $10 \mathrm{~kg}$ de massa corporal. Apresentando sangramento em mandíbula, agitação a manipulação, sendo necessário fazer $3 \mathrm{mg} / \mathrm{kg}$ de tramadol e $25 \mathrm{mg} / \mathrm{kg}$ de dipirona. Na primeira consulta ocorrida no dia 26 de setembro de 2019, a tutora 
relatou que o animal tinha sido atropelado. Quando feita anamnese a tutora citou que o animal tinha sido regatado da rua em janeiro de 2019, e possuía histórico de erliquiose canina. O animal foi encaminhado para o exame ultrassonográfico, onde constatou a presença de duas estruturas de parênquimas e formas iguais, sendo um deles apresentando descontinuidade do bordo em lobo cranial e parênquima heterogêneo sugerindo ruptura, também foi avaliada discreta quantidade de líquido livre abdominal assemelhando-se a hemorragia, as demais estruturas também foram avaliadas, porém, nada digno de nota (Figura 1).

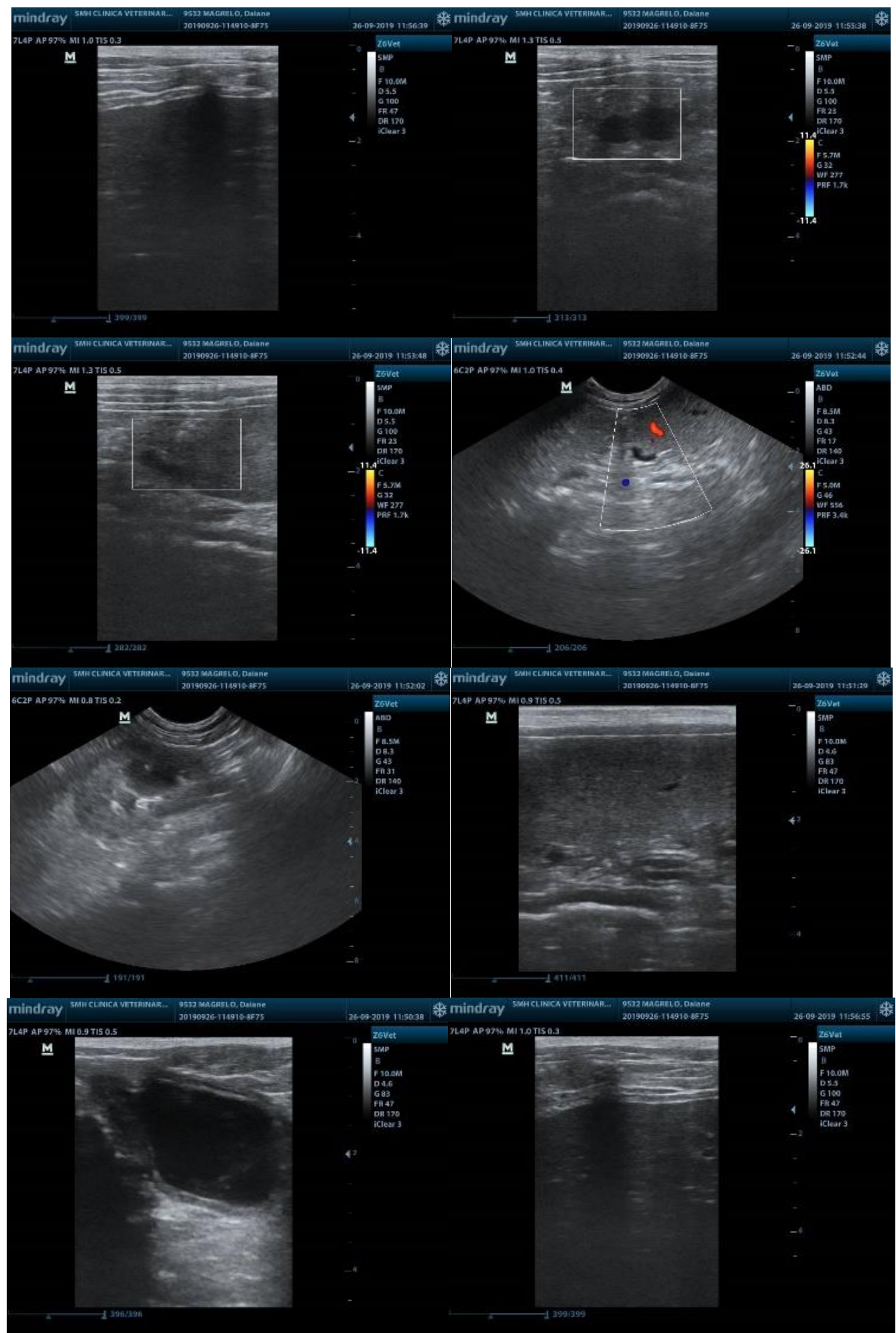

Figura 1. Canino macho, SRD, de 3 anos e 8 meses, imagem ultrassonográfica da região abdominal realizada no dia 26 de setembro de 2019. Presença de duas estruturas de igual parênquima e forma, sendo um deles apresentando descontinuidade do bordo em lobo cranial e parênquima heterogêneo. 
Foram realizados outros exames complementares como hemograma apresentando anemia normocítica normocrômica, hiperproteinemia, trombocitopenia (Tabela 1), na radiografia torácica não foram detectadas anormalidades.

Tabela 1. Resultados do hemograma, do canino, macho, sem raça definida, com 3 anos e 8 meses de idade, ao dar entrada no hospital escola veterinário.

\begin{tabular}{|c|c|c|c|c|}
\hline Eritrograma & & Resultado dia 26/09/2019 & & Referências \\
\hline Hemácias $\left(\mathrm{x} 10^{6} / \mu \mathrm{L}\right)$ & & 3,33 & & 5,5 a 8,5 \\
\hline Hemoglobina(g/dL) & & 7,8 & & 12,0 a 18,0 \\
\hline Hematócrito (\%) & & 23,6 & & 37,0 a 55,0 \\
\hline VCM (fL) & & 69,9 & & 60,0 a 77,0 \\
\hline$\overline{\mathrm{CHCM}(\%)}$ & & 33,2 & & 31,0 a 36,0 \\
\hline Eritrócitos Nucleados (/100 Leucócito & & 0 & & 0 a 10 \\
\hline Plaquetas (cels $/ \mu \mathrm{L})$ & & 90.000 & & 0.000 a 500.000 \\
\hline Leucograma & Resultados (cels $/ \mu \mathrm{l})$ & Referências & Valores relativos & Referências \\
\hline Leucócitos & 7.600 & 6.000 a 17.000 & & \\
\hline$\overline{\text { Basófilos }}$ & 0 & 0 a 100 & 0 & 0 a 1 \\
\hline Eosinófilos & 304 & 0 a 0 & 4 & 2 a 10 \\
\hline Mielócitos & 0 & 100 a 1.250 & 0 & 0 a 0 \\
\hline Metamielócitos & 0 & 0 a 0 & 0 & 0 a 0 \\
\hline Neutrófilos bastonetes & 0 & 0 a 300 & 0 & 0 a 3 \\
\hline Segmentados & 4.408 & 3.000 a 11.100 & $58-$ & 60 a 77 \\
\hline Linfócitos & 2.736 & $1.000 \mathrm{a} 4.800$ & $36+$ & 12 a 30 \\
\hline Monócitos & 152 & 150 a 1.350 & $2-$ & 3 a 10 \\
\hline Proteínas plasmáticas totais & $8,8+$ & 6,0 a 8,0 & & \\
\hline
\end{tabular}

O paciente foi então encaminhado para à sala de cirurgia, onde foi realizado uma esplenectomia somente do baço que possuía ruptura, consistindo em uma ampla incisão abdominal na linha média ventral para exposição adequada do baço, foi feito ligamento duplo e corte através de todos os vasos ao hilo esplênico com material de sutura absorvível, terminada a exérese do baço, ainda pôde-se verificar regeneração de tecido esplênico em região do mesentério (Figura 2). A síntese da parede abdominal foi realizada de maneira padrão.

No pós-operatório, o animal foi submetido a internação após retornar da cirurgia muito inconsciente apresentando demora ao acordar. No período da noite ele demonstrou-se mais ativo, com parâmetros vitais dentro da normalidade, mas indisposto a comer sozinho. $\mathrm{O}$ animal apresentava normuria, mas com presença de coloração escurecida. No período de internação o animal foi posto na fluidoterapia com ringer com lactato, macro gotas, $20,8 \mathrm{ml} / \mathrm{h}$, sendo administrado, dexametasona $(0,25 \mathrm{ml} / \mathrm{kg})$, dipirona $(0,6 \mathrm{ml} / 10 \mathrm{~kg})$, ondansetrona $(0,1-1 \mathrm{mg} / \mathrm{kg})$, por via intravenosa e transamin $(5-25 \mathrm{mg} / \mathrm{kg})$, agemoxi $(1$ $\mathrm{ml} / 10 \mathrm{~kg})$, tramadol (2 mg/kg), ranitidina (1-2 mg/kg), meloxicam $(0,1-0,2 \mathrm{mg} / \mathrm{kg})$ por via subcutânea. Após um período de 24 horas de internação o animal já se apresentava apto a continuar o tratamento com os tutores. Instituiu-se terapia por via oral com meloxicam $2 \mathrm{mg}$, administração de um comprimido SID por 4 dias, dipirona de uso humano administração de 10 gotas BID por via oral durante 5 dias, agemoxi cl 250ml administração de $3 / 4$ do comprimido BID por 14 dias, eritrós dog tabs administração de um comprimido BID por 30 dias e pomada para uso tópico na ferida cirúrgica vetaglós ${ }^{\circledR}$ (Sulfato Gentamicina + Sulfanilamida + Sulfadiazina + Ureia + Vitamina A - Palmitato) SID, após a prévia limpeza com solução fisiológica até a cicatrização e retirada dos pontos. Sendo orientado retornar no período de 15 dias para um novo hemograma e ultrassonografia abdominal para controle.

Quatro dias após, o animal retornou ao hospital veterinário e na anamnese quando indagada como o animal se apresentava a tutora informou que o animal apresentava fezes escuras, urina de coloração marrom, mas tendo como queixa principal, que o animal não estava deitando, demonstrava sinais de dor e um episódio de vômito na madrugada anterior a consulta clínica. Ao ser feito o exame físico do animal foi visto um quadro de dor aguda, edema ao redor do prepúcio, extravasamento de liquido pelo corte 
cirúrgico e apresentava tremor, a tutora alegou que o animal não respondia as administrações de dipirona e que também estava sendo falha a administração dos medicamentos prescritos.

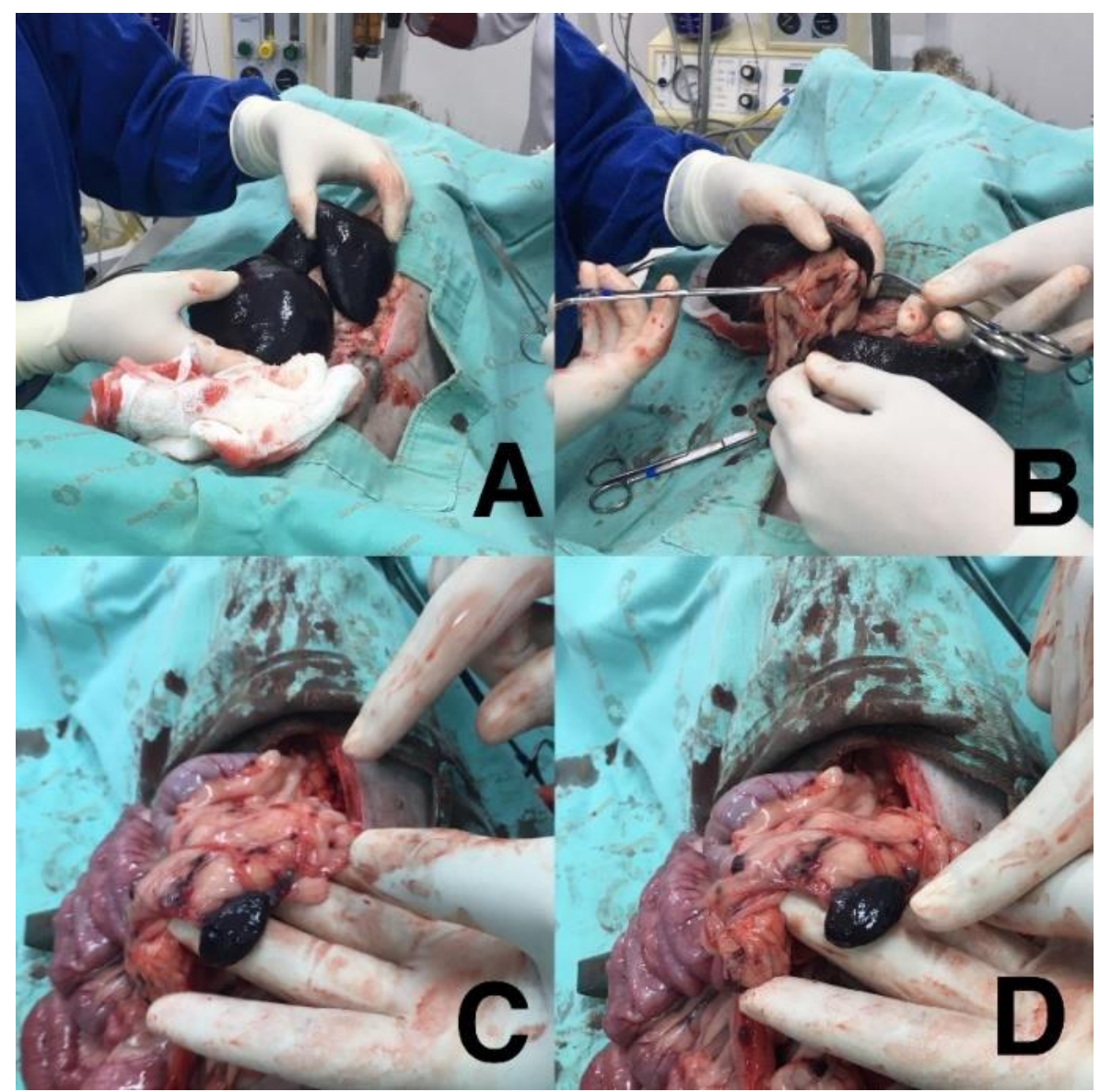

Figura 2. Canino macho, SRD, de 3 anos e 8 meses, imagem cirúrgica no qual pode-se observar: (A) Exposição dos dois baços após incisão abdominal. (B)- Ligamento duplo e corte transversal aos vasos ao hilo esplênico. (C e D) - Mesentério com evidência de tecido regenerativo esplênico.

Foi realizado um exame ultrassonográfico onde evidenciou peritonite severa com presença de líquido livre inflamatório, reação inflamatória em região de sutura, edema em bolsa escrotal. Sangramento característico de seroma, mas após esforço apresentou líquido mais sanguinolento. Foi solicitado internação do animal para controle da peritonite e dor aguda, hemograma (Tabela 2) e teste sorológico. $\mathrm{Na}$ internação o animal foi posto na fluidoterapia com ringer com lactato, macro gotas, $50 \mathrm{ml} / \mathrm{kg}$, e administrado no decorrer da internação metronidazol $(15 \mathrm{mg} / \mathrm{kg})$, ceftriaxona $(25-50 \mathrm{mg} / \mathrm{kg})$, dipirona $(0,6 \mathrm{ml} / 10 \mathrm{~kg})$, ondansetrona $(0,1-1 \mathrm{mg} / \mathrm{kg})$, por via intravenosa. E por via subcutânea ranitidina $(1-2 \mathrm{mg} / \mathrm{kg})$, meloxicam $(0,1-0,2 \mathrm{mg} / \mathrm{kg})$.

Tabela 2. Resultado do hemograma 3 dias após o procedimento cirúrgico e o início do tratamento, tendo como observação leucócitos e plaquetas com morfologia normal. Anisocitose e policromasia moderadas.

\begin{tabular}{lcc}
\hline Eritrograma & Resultado Dia 30/09/2019 & Referências \\
\hline Hemácias $\left(\mathrm{x} 10^{6} / \mu \mathrm{L}\right)$ & 3,11 & 5,5 a 8,5 \\
\hline Hemoglobina $(\mathrm{g} / \mathrm{dL})$ & 7,0 & 12,0 a 18,0 \\
\hline Hematócrito $(\%)$ & 21,6 & 37,0 a 55,0 \\
\hline VCM $(\mathrm{fL})$ & 70,0 & 60,0 a 77,0 \\
\hline CHCM $(\%)$ & 32 & 31,0 a 36,0 \\
\hline Eritrócitos Nucleados $(/ 100$ Leucócitos) & 0 & 0 a 10 \\
\hline Plaquetas $($ cels $/ \mu \mathrm{L})$ & 310.000 & 200.000 a 500.000 \\
\hline
\end{tabular}


Continuação Tabela 2. Resultado do hemograma 3 dias após o procedimento cirúrgico e o início do tratamento, tendo como observação leucócitos e plaquetas com morfologia normal. Anisocitose e policromasia moderadas.

\begin{tabular}{lcccc}
\hline Leucograma & Resultados $($ cels / $\mu \mathrm{l})$ & Referências & Valores relativos & Referências \\
\hline Leucócitos & 9.300 & 6.000 a 17.000 & & 0 a 1 \\
\hline Basófilos & 0 & 0 a 100 & 0 & 2 a 10 \\
\hline Eosinófilos & 837 & 0 a 0 & 4 & 0 a 0 \\
\hline Mielócitos & 0 & 100 a 1.250 & 0 & 0 a 0 \\
\hline Metamielócitos & 0 & 0 a 0 & 0 & 0 a 3 \\
\hline Neutrófilos bastonetes & 0 & 0 a 300 & 0 & 60 a 77 \\
\hline Segmentados & 6.324 & 3.000 a 11.100 & $58-$ & 3 a 30 \\
\hline Linfócitos & 1.953 & 1.000 a 4.800 & $36+$ & 3 a 10 \\
\hline Monócitos & 186 & 150 a 1.350 & $2-$ & \\
\hline Proteínas plasmáticas totais $(\mathrm{g} / \mathrm{dL})$ & & & &
\end{tabular}

Após 24 horas de internação o animal já apresentava apto a ser liberado para continuar o tratamento com a tutora. Através do resultado do teste sorológico feito com o plasma sanguíneo do animal, foi confirmado a presença de Ehrlichia canis e Anaplasma platys, sendo prescrito Doxiciclina $10 \mathrm{mg} / \mathrm{kg}$ SID, por via oral durante 28 dias. Três dias após o animal retornou para uma reavaliação clínica onde apresentava se mais ativo e confortável, apetite preservado, sem êmese. O quadro de hemorragia pela ferida cirúrgica cedeu por completo, parâmetros vitais dentro da normalidade, mucosas normocoradas apresentando um ótimo estado geral.

Sete meses após a cirurgia (Figura 3) o animal se apresentava sadio, ativo, com aumento do peso corporal.

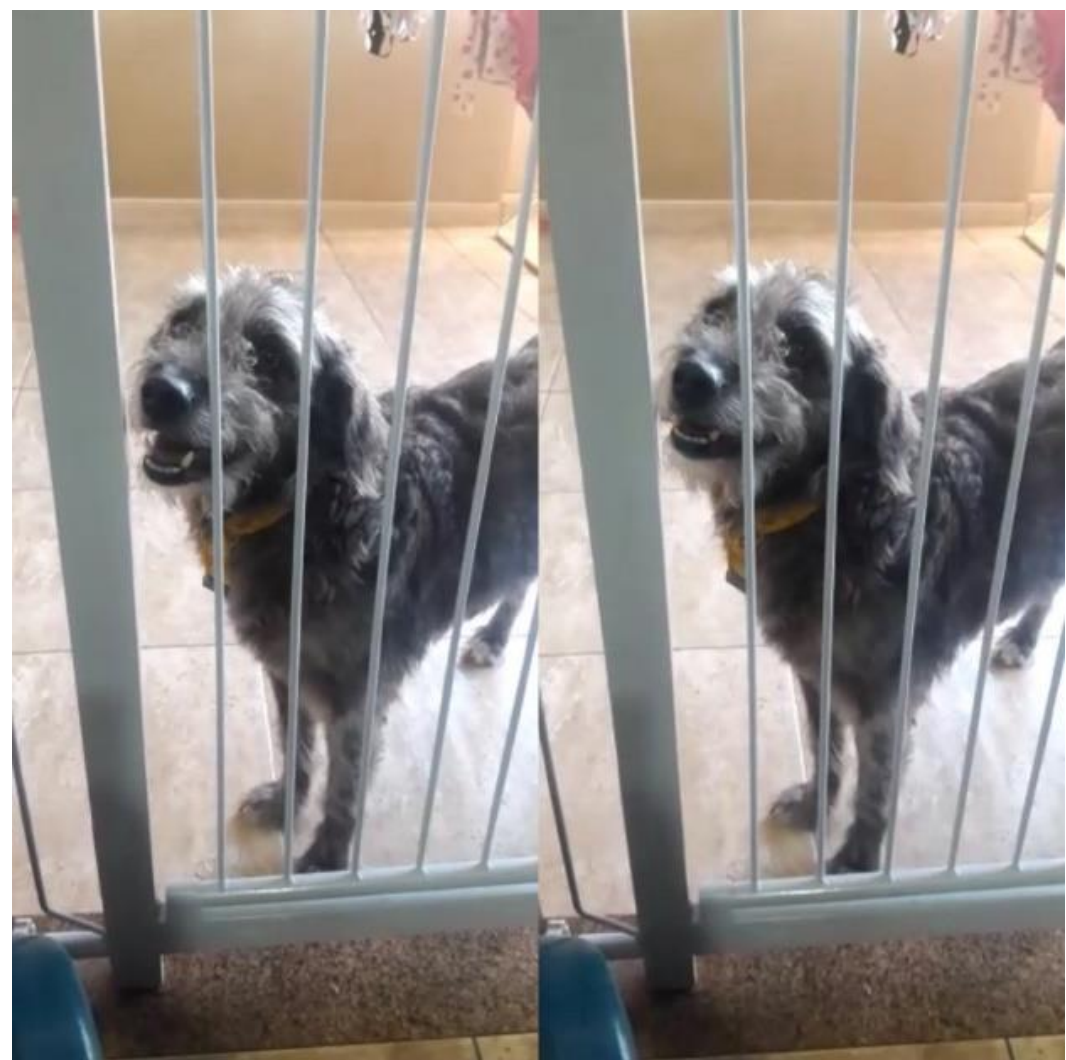

Figura 3. Canino macho, SRD, 4 anos de idade, 7 meses após esplenectomia total e tratamento para Ehrlichia canis e Anaplasma platys, se apresentando sadio, ativo, com aumento do peso corporal.

\section{Discussão}

Segundo Slatter (1998) a sintomatologia de uma alteração esplénica geralmente são inespecíficos, e descritas muitas das vezes como provenientes de uma doença primária o que não corrobora com o 
presente relato em que tratou-se de um trauma devido ao atropelamento sofrido pelo paciente (Aster, 2005), a ruptura esplénica em cães não é usual, porem ao ocorrer provem de trauma; atropelamento ou outro acidente. Sendo assim o animal descrito apresentava ruptura de baço devido ao trauma ocasionado por um atropelamento como descrito em literatura. Além da alteração esplênica (Slatter, 1998), também podem ser encontradas alterações hematológicas implícitas manifestando na maioria dos casos alterações a nível de contagem eritrócitos sendo mais frequente a presença de anemias, como visto presente no primeiro hemograma.

O uso de exames complementares é de suma importância visto que eles vão proporcionar um direcionamento tanto na conduta operatória quanto na pós-operatória, sendo um deles a ultrassonografia que por ser um método de imagem, ela possibilita a avaliação de anormalidades e alterações na ecogenicidade e ecotextura do parênquima (Maronezi et al., 2015). Ela possibilitou reconhecer que o animal possuía dois órgãos esplênicos de estruturas de parênquimas e formas iguais, sendo um deles lesado pelo trauma sofrido o que possibilitou a escolha da esplenectomia total. De acordo com (Fossum, 2014) a esplenectomia ela é justificável caso ocorram falhas na terapia medicamentosa ou efeitos adversos, porem este dado não fundamenta o animal descrito, pois a esplenectomia realizada se deu pelo fato do paciente possuir dois órgãos sendo preferível a retirada do órgão lesado, preservando somente o órgão sadio. Não foram encontrados relatos publicados dessa alteração morfológica em animais, porem há relatos em humanos onde se afirma que de 6 a $10 \%$ da população tem um baço a mais e que a presença do segundo órgão não costuma causar problemas, sendo ele de um tamanho menor do que originalmente seria se assemelhando a um feijão e uma maçã (Junqueira \& Carneiro, 2013), o que não corrobora com o animal descrito nesse relato, pois o segundo baço se apresentava de tamanho e forma semelhante à forma normal de um baço de espécie canina. No caso relatado a técnica cirúrgica efetuada consiste em um procedimento padrão de esplenectomia (Fossum, 2014).

Na esplenectomia em cães (Fossum, 2014) ainda relata que diversas complicações podem ocorrer sendo a principal hemorragia e subsequentes são as sépticas, por alteração de fluxo sanguíneo e infecções subclínicas devido à hemoparasitas. Todavia o paciente não apresentou hemorragia, mas quatro dias após a cirurgia o animal foi submetido ao exame complementar sorológico onde se comprovou a presença de Ehrlichia canis e Anaplasma platys. Fossum (2014) ainda cita que essas enfermidades podem se tornar óbvias após esplenectomia.

Os sinais clínicos mais comumente visualizados em cães com alterações esplênicas normalmente resultam em vómito, fraqueza, anorexia, colapso, perda peso, distensão abdominal, polidipsia e poliúria (Slatter, 1998). Todavia, de todos os sinais descritos, o único visto no paciente foi a presença de vomito quatro dias após a conduta cirúrgica, o que pode ter relação com o fato de ser um sinal clínico associado ao quadro de peritonite severa (Zimmermann et al., 2006) que o animal em questão apresentou. Como indicado por Zimmermann et al. (2006) tratamento da peritonite secundária consiste em estabilização hemodinâmica, imunológica e metabólica do animal associando a antibioticoterapia para o controle do foco infeccioso e remoção da causa. No presente relato o cão foi tratado inicialmente com metronidazol e ceftriaxona após ser colocado na fluidoterapia, corroborando com Zimmermann et al. (2006).

No tratamento da erliquiose canina e da anaplasmose foi prescrito Doxiciclina na dose de $5 \mathrm{mg} / \mathrm{kg}$, via oral, BID, durante 28 dias, sendo o fármaco de eleição, com recuperação clínica em 24-72h (Brandão, 2010). Quatro dias após o início do tratamento o animal já se apresentava mais ativo, confortável, sem presença de vomito e com os parâmetros vitais dentro da normalidade, apresentando um ótimo estado geral. Com isso o relato evidencia uma baixa casuística, ainda que na rotina clínica cirúrgica, a esplenectomia total por ruptura de baço seja descrita em literatura, um animal apresentando 2 órgãos esplênicos não é relativamente comum.

\section{Conclusão}

Em virtude dos fatos mencionados o desempenho da esplenectomia em um paciente com dois baços, teve sucesso apesar das alterações obtidas no pós-operatório, sendo possível acompanhar a recuperação efetiva do paciente junto à terapêutica instituída. 


\section{Referências bibliográficas}

Aster, J. C. (2005). Disease of white blood cells, lymph nodes, spleen, thymus. Robbins and Cotran Pathologic Basis of Disease, 679, 702-705.

Brandão, L. (2010). Hemoparasitoses em cães e gatos: aspectos clínicos e laboratoriais. MERIAL Saúde Animal. Fossum, T. W. (2014). Cirurgia de pequenos animais (4th ed., Vol. 1). Elsevier Brasil.

Junqueira, L. C., \& Carneiro, J. C. (2013). Histologia Básica (12 ed.). Guanabara Koogan.

Kisseberth, W. C., \& McEntee, M. C. (2008). Doenças do baço. In B. S.J. \& S. R.G. (Eds.), Manual Saunders Clínica de Pequenos Animais (pp. 277-287). Roca.

Maronezi, M. C., Feliciano, M. A. R., Crivellenti, L. Z., Borin-Crivellenti, S., Silva, P. E. S., Zampolo, C., Pavan, L., Gasser, B., Simões, A. P. R., \& Maciel, G. S. (2015). Spleen evaluation using contrast enhanced ultrasonography and Doppler in dogs with subclinical ehrlichiosis. Arquivo Brasileiro de Medicina Veterinária e Zootecnia, 67(6), 1528-1532. DOI: https://doi.org/10.1590/1678-4162-7941

Petroianu, A., Pereira, T. C., Oliveira, T. A. N., \& Barbosa, A. J. A. (2004). Assessment of different conservative methods of the spleen for treatment of splenic incisional lesion in the dog. Revista Do Colégio Brasileiro de Cirurgiões, 31(6), 364-367.

Slatter, D. H. (1998). Manual de cirurgia de pequenos animais (Vol. 2). Manole São Paulo.

Stedile, R. (2007). Esplenectomia em cães: comparação entre os acessos laparoscópico e convencional. Universidade Federal do Rio Grande do Sul.

Zimmermann, M., Raiser, A. G., Mazzanti, A., Lopes, S. T. dos A., \& Salbego, F. Z. (2006). Peritonitis in dogs. Ciência Rural, 36(5), 1655-1663. DOI: https://doi.org/10.1590/s0103-84782006000500052

\section{Histórico do artigo:}

Recebido: 21 de julho, 2020.

Aprovado: 18 setembro, 2020.

Disponível online: 9 de novembro, 2020.
Licenciamento: Este artigo é publicado na modalidade Acesso Aberto sob a licença Creative Commons Atribuição 4.0 (CC-BY 4.0), a qual permite uso irrestrito, distribuição, reprodução em qualquer meio, desde que o autor e a fonte sejam devidamente creditados. 